\title{
Characteristics and quality of genetics and genomics mobile apps: a systematic review
}

\author{
Divya Talwar $\mathbb{B}^{1,2} \cdot$ Yu-Lyu Yeh ${ }^{1} \cdot$ Wei-Ju Chen ${ }^{1} \cdot$ Lei-Shih Chen $\mathbb{D}^{1}$
}

Received: 26 July 2018 / Revised: 17 January 2019 / Accepted: 24 January 2019 / Published online: 26 February 2019

(c) European Society of Human Genetics 2019

\begin{abstract}
Mobile applications (apps) have been increasingly utilized to access the latest and abundant information related to genetics/ genomics for resources, risk assessments, and individualized recommendations. Nevertheless, the number and quality of the current apps in genetics/genomics remain unknown. Thus, in this review, we aimed to identify existing genetic/genomic apps, summarize their characteristics, and examine their quality. A systematic search of genetics/genomics apps was conducted on Apple Store and Google Play. We adapted a validated evaluation scale, Mobile App Rating Scale (MARS), to examine the quality of genetics/genomics apps. Eighty-eight genetics/genomics apps, with the cost ranging from free to $\$ 49.99$, formed the final sample. Findings showed that the majority of the apps had reference/resource as a feature (95.5\%), had health professional students as the target audience (86.4\%), and did not focus on specific diseases (78.5\%). Only $21.6 \%$ of the apps were developed by reliable or authoritative agencies, and the apps' overall quality was slightly above average based on the criteria of the MARS. Therefore, while genetics/genomics mobile apps might be useful resources, their quality still needs improvement, especially with respect to the credibility and evidence-based items of app information as well as the customization items of app engagement; caution must be taken when using those apps.
\end{abstract}

\section{Introduction}

Global smart phone usage has increased tremendously in recent years. As of 2016, there was an estimate of 140 billion mobile application (or app) downloads via Apple Store [1] and almost 65 billion mobile app downloads through Google Play (Android) [2]. A 2012 nationwide survey showed that approximately one-fifth of American mobile phone users had downloaded a healthrelated app [3], and such prevalence may have increased to $58 \%$ which was found in a more recent study in 2015 [4].

Currently, the mobile health industry is only loosely and ambiguously regulated by authorities, such as the Food and

Supplementary information The online version of this article (https:// doi.org/10.1038/s41431-019-0360-2) contains supplementary material, which is available to authorized users.

Lei-Shih Chen

lacechen@tamu.edu

1 Department of Health and Kinesiology, Texas A\&M University, College Station, TX, USA

2 The Children's Hospital of Philadelphia, Philadelphia, PA, USA
Drug Administration (FDA) in the United States [5, 6], Medical Devices Directive (MDD) of the European Union [7-9], and Medicines and Healthcare products Regulatory Agency (MHRA) in the United Kingdom [9, 10]. In addition to the regulation concern, common faults of apps include poor aesthetics, inaccurate content and information, fluctuating or spotty functionality, and an inability to engage users [11].

There are three levels of mobile health apps in the United States [6]. Level 1 health apps are marketed as medical devices, such as apps that can help determine if a patient's mole is cancerous [12], which require formal FDA review and approval before marketing and dissemination. Level 2 apps are "wellness" apps (e.g., food tracking, step counters, and lifestyle coaches). Because those apps are not life-threatening, FDA approval is not needed [6]. Level 3 apps encompass all the apps that are neither Level 1 nor Level 2, and they are exempt from the FDA approval. Some of the apps could be medical devices but are not marketed as life-saving avenues and/or medical devices [6].

An app regulation system also takes place in Europe. Apps considered as medical devices (e.g., those used in medical diagnosis or treatment) must pass the conformity 
assessment and obtain a "Conformité Européenne" (CE) marking. Such apps are regulated by the MDD of the European Union and MHRA in the United Kingdom [7-10]. Criticism, however, has risen in regards to the possibly obscure definition of a medical device and the insufficient lists of the apps with the CE marking released by the authorities. Furthermore, there is a disagreement in the regulations for health apps that are not categorized as medical devices [10].

With the completion of the Human Genome Project in 2003 [13], genomics became a rapidly evolving field with new advances in genetic testing and information every year. Mobile apps are increasingly utilized for accessing latest and abundant information related to genomics. Functions and benefits of genetics and genomics mobile apps include educational resources, risk assessments for diseases, and individualized recommendations [14-17]. While researchers have evaluated mobile apps addressing health topics, such as cancer [18], diabetes [19, 20], depression [21], and physical activity [22], the number and quality of existing apps in genetics and genomics remain unknown. Moreover, along with the insufficient regulations by the authorities (e.g., the FDA, MDD, and MHRA), potential problems with those apps may include inaccurate information, unreliable references and resources, misguided genetic test results, and inappropriate genetic algorithm calculations for risks of various diseases. To maximize the benefits and minimize the potential risks arising from genetics and genomics apps, in this systematic review, we sought to examine the number, characteristics, and quality of existing mobile apps in genetics and genomics.

\section{Materials and methods}

\section{Apps search and screening}

We utilized a systematic review framework [23] for the app searching and screening as well as data extraction and coding. To ensure a comprehensive inclusion of all mobile apps in genetics and genomics, we included both Apple and Android apps - the major app platforms - by searching apps on both Apple Store and Google Play until October 14, 2017. Figure 1 indicates the app search, screening, and selection process. Specifically, we screened genetics and genomics mobile apps in three steps. In step one, we searched apps using the keywords: "gene," "genes," "genetic," "genetics," "genome," "genomic," "genomics," "family history," and "family health history." In step two, we then screened the apps for duplicates, relevance, and languages. We also assessed the eligibility of the apps based on the inclusion and exclusion criteria through the initial screening by reviewing their description before downloading and via

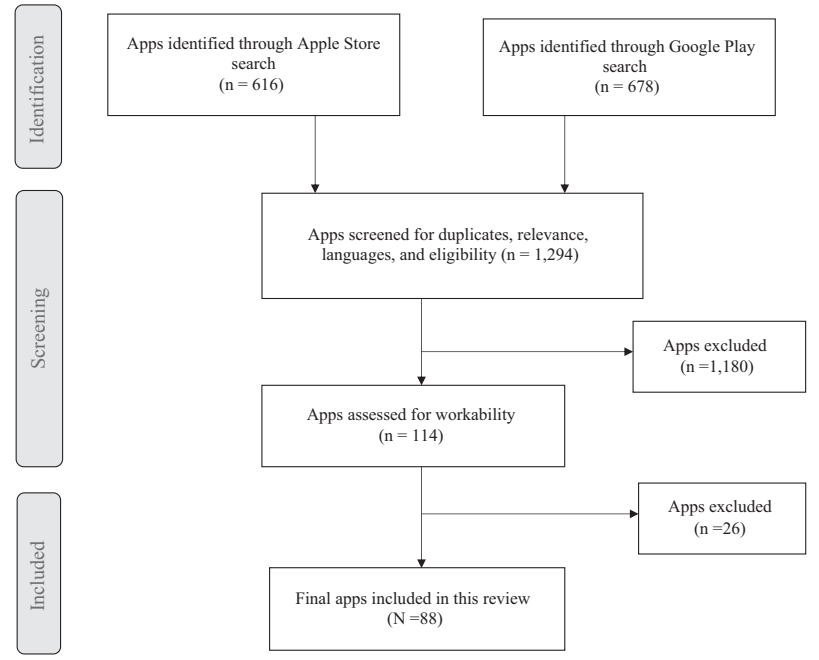

Fig. 1 App search, screening, and selection procedure

the second screening for the subsequent downloaded apps. The inclusion criteria were apps focused on genetics or genomics, with applied or clinical context, and in English. The exclusion criteria included: (1) apps that outlined conference programs; (2) apps that advertised and/or provided scientific journals and books; (3) apps focusing on animals or plants; (4) non-genetics/genomics focused apps; (5) apps without medical or clinical applications; (6) apps from private companies which required an uploaded genetic profile in order to gain access; (7) apps designed for only high school students (determined based on the app descriptions); and (8) non-English apps. Moreover, some apps had both a free version with basic features and a paid version with full features; in such cases, we reviewed the paid version for this study. In the last step, we downloaded the apps to examine potential issues with workability, such as if apps would crash while downloading and lack of compatibility with the current operation systems. For instance, at the time of the app search, Apple Inc. devices used the iOS mobile operating system version 11 (iOS 11). Some apps that were created earlier were not compatible with iOS 11 preventing us from downloading and/or opening the apps. In such cases, we excluded those apps.

\section{Data extraction and coding}

We extracted and examined the app data in terms of their characteristics, including the name, version, developer, type of developer (e.g., commercial/private company, university/ hospital based, professional organization, government, and non-governmental organization [NGO]), operating system (i.e., iPhone, iPad, and/or Android), app description, app features (e.g., characterized as reference/resource, clinical tool, gaming, avenue for lifestyle change recommendations, provision of genetic test results, and quizzing), technical 
Table 1 Descriptive statistics of the main characteristics of the genetics/genomics apps

\begin{tabular}{|c|c|c|c|}
\hline Characteristic & Category & $\begin{array}{l}n / \text { Mean } \\
(\mathrm{SD})\end{array}$ & $\% /$ Range \\
\hline \multirow[t]{6}{*}{ Type of developer } & $\begin{array}{l}\text { App created by commercial/private companies or } \\
\text { individuals }\end{array}$ & 49 & $55.7 \%$ \\
\hline & App created by universities or hospitals & 12 & $13.6 \%$ \\
\hline & App created by non-governmental organizations & 3 & $3.4 \%$ \\
\hline & App created by government & 2 & $2.3 \%$ \\
\hline & App created by professional organizations & 2 & $2.3 \%$ \\
\hline & $\begin{array}{l}\text { Insufficient information/not mentioned presented in } \\
\text { the app content/unknown }\end{array}$ & 20 & $22.7 \%$ \\
\hline \multirow[t]{3}{*}{ Operating system } & iPad & 57 & $64.8 \%$ \\
\hline & iPhone & 52 & $59.1 \%$ \\
\hline & Android & 47 & $53.4 \%$ \\
\hline \multirow[t]{6}{*}{ App feature(s) } & Reference/resource & 84 & $95.5 \%$ \\
\hline & Clinical tool & 16 & $18.2 \%$ \\
\hline & Gaming & 11 & $12.5 \%$ \\
\hline & Lifestyle change recommendations & 8 & $9.1 \%$ \\
\hline & Provision of genetic test results & 6 & $6.8 \%$ \\
\hline & Quizzing & 6 & $6.8 \%$ \\
\hline \multirow[t]{3}{*}{ Technical aspects of app } & Needing web access to function & 85 & $96.6 \%$ \\
\hline & Permitting password protection & 22 & $25.0 \%$ \\
\hline & $\begin{array}{l}\text { Allowing sharing on Facebook/Twitter/having an app } \\
\text { community }\end{array}$ & 16 & $18.2 \%$ \\
\hline \multirow{2}{*}{$\begin{array}{l}\text { Focus on specific } \\
\text { disease }\end{array}$} & Yes & 20 & $22.7 \%$ \\
\hline & No & 68 & $78.5 \%$ \\
\hline \multirow[t]{5}{*}{ Target audience(s) } & Health professional students & 76 & $86.4 \%$ \\
\hline & $\begin{array}{l}\text { Health professionals/researchers/medical } \\
\text { professionals }\end{array}$ & 49 & $55.7 \%$ \\
\hline & General population & 31 & $35.2 \%$ \\
\hline & Patients & 9 & $10.2 \%$ \\
\hline & Parents & 2 & $2.3 \%$ \\
\hline Customer rating $^{\mathrm{a}}$ & & $4.0(0.5)$ & $3.0-5.0$ \\
\hline Cost & & $\begin{array}{l}\$ 2.18 \\
(7.85)\end{array}$ & $\$ 0-\$ 49.99$ \\
\hline
\end{tabular}

${ }^{a}$ Only 54 apps (61.4\%) had customer ratings aspects (e.g., requiring web access to function, permitting password protection, and allowing sharing on social media such as Facebook, Twitter, or an app community), whether or not the app focuses on a specific disease, target audience, customer rating, and cost. The data were mainly based on the descriptions provided in the apps (e.g., apps description included their target audiences and app features).

\section{Quality of apps: the Mobile App Rating Scale (MARS)}

We adapted the MARS to examine the quality of the apps included in this study (Supplementary Appendix 1) [24]. The MARS is a well-known, validated scale developed by the researchers at Queensland University of Technology in Australia to measure the quality of mobile health apps. The
MARS has four categories: app classification, objective app quality, subjective app quality, and app specific items $[11,24,25]$. To be consistent with past studies $[11,25]$ and to assess the quality of apps objectively, this study included two categories from the MARS: app classification and objective app quality. The app classification elicited descriptive information of the apps, such as the type of developer, target audience, and cost, which are summarized in Table 1. The objective app quality scale (MARS score) measures the quality of apps using four scales (i.e., engagement, functionality, aesthetics, and information) with 19 five-point items, each scoring from one to five.

The engagement scale, measuring the extent to which the apps are fun, interesting, interactive, and appropriate for the target audience, consists of five items: entertainment, 
interest, customization, interactivity, and target group. The four items in the functionality scale, designed to assess the user-friendliness of the apps and app functions, are performance, ease of use, navigation, and gestural design. There are three items in the aesthetics scale: layout, graphics, and visual appeal, which examine the degree to which the apps are aesthetically pleasing. Information scale, assessing the accuracy, credibility, quality, and quantity of the app information, consists of seven items: accuracy of app description, goals, quality of information, quantity of information, visual information, credibility, and evidence base. Each scale score is computed using the mean of the item scores (ranging from one to five).

The overall MARS score is the average of the four scale scores (i.e., engagement score, functionality score, aesthetics score, and information score). The range of the MARS score is also from one to five, with a higher score representing better app quality. Two authors of this manuscript (D.T. \& Y.L.Y.) independently assessed all apps for the interrater reliability of the MARS score. We examined the interrater reliability using the Intraclass correlation coefficient (ICC) through two-way random-effects model, which was commonly used in past studies for assessing the interrater reliability of the MARS score [26-28]. The ICC was 0.91 indicating an excellent interrater reliability. Moreover, the two authors (D.T. \& Y.L.Y.) discussed any disagreement for the MARS score rating and reached the consensus.

\section{Results}

We identified 1294 relevant apps; of which, 616 were from Apple, and 678 were from Android. After the screening for duplicates, relevance, languages, and eligibility criteria, 1180 apps were excluded. We downloaded the remaining 114 apps on iPhone, iPad, and/or Android devices. Twentysix apps were excluded due to lack of workability. For example, some apps could not be opened after download (e.g., MyGene), and several apps were not updated to the latest iOS systems on Apple Stores (e.g., Genesys Dysmorphology Database, My Cancer Genome, and Know: BRCA). The final sample included in this review consisted of 88 genetics/genomics apps (Fig. 1).

\section{Characteristics of the included genetics/genomics apps}

Table 1 shows the summary and main characteristics of the included genetics/genomics apps. Supplementary Table S1, which was based on the MARS shown in Supplementary Appendix 1, contains detailed information of the apps, such as the name, developer, type of developer, operating system, brief description, app features, technical aspects, specific disease focus, target audience, customer rating, and cost for both the basic and upgraded versions.

Of the total 88 genetics/genomics apps, slightly more than half $(n=49 ; 55.7 \%)$ were created by commercial/private companies or individuals. Less than a quarter of the apps were created by reliable or authoritative agencies, including universities or hospitals $(n=12 ; 13.6 \%)$, NGOs $(n=3 ; 3.4 \%)$, professional organizations such as the American College of Medical Genetics and Genomics (ACMG) $(n=2 ; 2.3 \%)$, and government agencies such as the National Institute of Health (NIH) and Centers for Disease Control and Prevention (CDC) $(n=2 ; 2.3 \%)$. Most apps were applicable on multiple platforms, including $\mathrm{iPad}(n=57 ; 64.8 \%)$, iPhone $(n=52 ; 59.1 \%)$, and Android $(n=47 ; 53.4 \%)$.

There were six types of features for genetics/genomics apps: reference/resource, clinical tool, gaming, lifestyle change recommendations, provision of genetic test results, and quizzing. Twenty-eight apps (31.8\%) had two types of features, and 11 apps (12.5\%) included more than two features. Reference or resource was an app feature in a majority of the genetics/genomics apps $(n=84 ; 95.5 \%)$. Those reference or resource apps included general information and/or tutorial regarding genetics, genetic testing, specific genetic disorders, and others. For example, an app titled Baby's First Test, created by the Genetic Alliance, serves as a reference or resource app for various audiences, such as the general public, health professionals, health professional students, and parents, to provide a quick reference guide for newborn screening information. In addition to the reference/resource apps, 16 apps (18.2\%) were designed as clinical tools to provide clinical diagnosis, assist in clinical decision-making, develop treatment algorithms for genetic diseases, perform pedigree analysis, and conduct risk assessment for health professionals. For example, the app Face2Gene can detect phenotypes from facial pictures and further evaluate and compare phenotypical variations for facial dysmorphology disorders. Moreover, gaming was a feature for 11 apps (12.5\%). For example, Cootie Genetics!, an interactive app developed by the University of Arizona Biotech Project, helps health professional students and the general public learn about basic genetics concepts such as the Mendelian laws of inheritance in the form of a game. Some apps $(n=8 ; 9.1 \%)$, which were often linked to direct-to-consumer (DTC) genetic test kits, were intended to provide lifestyle change recommendations mainly for the general public. Genomapp: Squeeze your DNA, for instance, is a commercial app, which provided lifestyle suggestions based on the genetic test results from three DTC genetic testing companies (i.e., 23andme, Decodeme, and Navigenics). Few apps $(n=6 ; 6.8 \%)$ included the provision of genetic test results as an app's function. For example, Invitae Corporation 
developed Invitae Family History Tool to provide a platform for genetic test orders and reports for testing results. Additionally, six apps (6.8\%) had quizzing as an app feature. For instance, Gene Tutor is a genetics quizzing and reference app designed for students taking the course "Molecular and Mendelian Genetics" at Columbia University.

Regarding the technical functions (i.e., requiring web access to function, password protection, sharing to social media-mainly Facebook and Twitter), almost one-third of the apps $(n=32 ; 36.4 \%)$ had multiple technical functions. Moreover, the majority of the apps $(n=85 ; 96.6 \%)$ required a web access to function. Only approximately one quarter of the apps $(n=22 ; 25.0 \%)$ had protective features like password protection. Some apps $(n=16 ; 18.2 \%)$ had a social network function allowing users to share messages on Facebook/Twitter or an app community. For example, customers, who use Framingham CRP Score - Reynold's Risk, could share their risks of cardiovascular disorders on Twitter with others.

Less than a quarter of the apps $(n=20 ; 22.7 \%)$ focused on specific chronic or genetic disorders. The targeted chronic disorders included cancer $(n=7)$, familial hypercholesterolemia $(n=1)$, cardiovascular disease $(n=$ $1)$, reproductive health $(n=1)$, and chronic obstructive pulmonary disease $(n=1)$. For example, myRisk, developed by the Myriad Genetics, Inc, focuses on eight hereditary cancers (i.e., breast, colorectal, ovarian, endometrial, gastric, pancreatic, melanoma, and prostate cancer). For those apps targeting genetic disorders, they addressed cystic fibrosis $(n=1)$, Prader Willi $(n=1)$, Amyloidosis $(n=1)$, Alzheimer's disorder $(n=1)$, inborn errors of metabolism $(n=1)$, intellectual disability disorders $(n=1)$, and other rare genetic disorders $(n=3)$. For instance, the Cold Spring Harbor Laboratory developed Gene Screen, which focuses on recessive genetic diseases among the Ashkenazi Jewish population, as a reference/resource app for health professionals, students, and researchers.

Almost three quarters of the genetics/genomics apps had multiple target audiences $(n=60 ; 68.2 \%)$. Most of the apps were for health professional students $(n=76 ; 86.4 \%)$. For instance, a commercial company created Genetics 101 by GoLearningBus which is a reference for basic genetic concepts for health professional students. Furthermore, more than half of the genetics/genomics apps aimed to reach health professionals, researchers, or medical professionals $(n=49 ; 55.7 \%)$. As an example, Genetic Counseling Aids, an app developed by Greenwood Genetic Center, is an educational reference app for genetic counselors, educators, and other genetic healthcare professionals for patient education and student instruction. Some genetics/ genomics apps target patients $(n=9 ; 10.2 \%)$. For example, TapGenes Family Health History, developed by TapGenes
Inc., is an interactive app that could allow patients to save their family health history information to discuss with physicians during a medical appointment. The target population for several apps $(n=31 ; 35.2 \%)$ was the general population. Atlantic Health System developed My Family Health History app, for instance, to provide an online portal system for lay people to personally record their family health history, calculate genetic risks for chronic diseases such as diabetes, breast cancer, and colon cancer, and access genomics educational resources. Only two (2.3\%) apps were for parents. As an example, Prader Willi World, a resource and reference app for parents of children with Prader Willi, allows parents to connect with each other and share app content on Facebook and Twitter.

The customer ratings, which were scored by the genetics/ genomics app users on Apple Store or Google Play, provide information about the popularity, strengths, and weaknesses of the apps. Over one-third of the apps $(n=34 ; 38.6 \%)$ did not have a customer rating due to the lack of an adequate number of reviewers. The other apps $(n=54 ; 61.4 \%)$ had an average customer rating of 4.0/5.0 (range $=3.0-5.0$ ). Regarding the cost for the genetics/genomics apps, the price ranged from free to $\$ 49.99$. The apps with the highest cost (\$49.99) were Genetic Counseling Aids and PediaGene: $A A P$ (full version). Most of the apps were free; 66 apps $(75.0 \%)$ were completely free, and 8 apps $(9.1 \%)$ had a free basic version and charged more for a full version $(\$ 2.99$ \$49.99).

\section{MARS ratings}

The Supplementary Table S2 outlines the MARS scores for the 88 genetics/genomics apps. As each scale of the MARS (i.e., engagement, functionality, aesthetics, and information) was scored using a five-point scale, a score of one would mean inadequate app quality, while a score of five represents excellent app quality. Table 2 shows the means, standard deviations, minimums, and maximums of the MARS ratings. The average MARS score for all included apps in our study was 3.78 ( $\mathrm{SD}=0.58)$, ranging from 2.42 to 4.79 , which was slightly above the average based on the MARS criteria [5, 27]. The top apps with the five highest MARS scores were My Genetics Compass (4.79/5.00), Prader Willi World (4.78/5.00), Invitae Family History Tool (4.75/5.00), Power of Minus Ten - Cells and Genetics (4.64/ 5.00), Gene Tutor (4.63/5.00), and DNA Play (4.63/5.00). Conversely, the apps that had the five lowest MARS scores were Genetic Disorder (2.42/5.00), All about Genes and Genetics (2.73/5.00), Genetics (2.73/5.00), GenCode (2.85/ 5.00), AP Biology Hardy-Weinberg Spreadsheet Tutorial (2.86/5.00), and Genome Genius (2.88/5.00).

We used the four scale scores (i.e., engagement score, functionality score, aesthetics score, and information score), 
Table 2 Descriptive statistics of the MARS ratings of the 88 genetics/genomics apps

\begin{tabular}{lllll}
\hline & Mean & Standard deviation & Minimum & Maximum \\
\hline App engagement score & 3.69 & 0.81 & 2.20 & 5.00 \\
App functionality score & 4.14 & 0.66 & 2.75 & 5.00 \\
App aesthetics score & 3.70 & 0.85 & 1.33 & 5.00 \\
App information score & 3.61 & 0.68 & 1.40 & 4.80 \\
Overall MARS score & 3.78 & 0.58 & 2.42 & 4.79 \\
\hline
\end{tabular}

MARS Mobile App Rating Scale each ranging from one to five, to calculate the MARS scores. The average engagement score for all reviewed apps was $3.69(\mathrm{SD}=0.81)$ with a range of $2.20-5.00$, indicating slightly above average in this aspect. The item scores of engagement from the lowest to the highest were as follows: customization (2.43/5.00), entertainment (3.91/5.00), interest (3.98/5.00), interactivity (3.99/5.00), and target group (4.15/5.00). Moreover, the average functionality score for the reviewed apps was $4.14(\mathrm{SD}=0.66)$ ranging from 2.75 to 5.00, suggesting good app functionality overall. The scores for the functionality items, listed from the lowest to highest, were app performance (4.01/5.00), gestural design (4.11/5.00), ease of use (4.17/5.00), and navigation setting (4.25/5.00). Additionally, the average aesthetics score for the apps was $3.70(\mathrm{SD}=0.85)$ ranging from 1.33 to 5.00 , with the item scores of 3.43/5.00, 3.52/5.00, and 4.14/5.00 for visual appeal, graphics, layout, respectively. Lastly, the average app information score was $3.61(\mathrm{SD}=0.68)$ ranging from 1.40 to 4.80 , and its average item scores were 2.08/5.00 for evidence base, 2.66/5.00 for credibility, 3.57/ 5.00 for quality of information, 3.62/5.00 for quantity of information, 4.16/5.00 for accuracy, 4.17/5.00 for visual information, and 4.29/5.00 for goal setting.

\section{Discussion}

To the best of our knowledge, this is the first study that aimed to identify the existing genetics/genomics apps and further evaluate their quality using the MARS. As the first in this area of research, we thoroughly described the characteristics of the genetics/genomics apps to provide a better understanding of their features and functions. This topic is important for health professionals in the genomics field as various professional organizations and researchers recommend health professionals to acquire the latest genomic information by using information technologies [29-31]. For example, the National Coalition for Health Professional Education in Genetics (NCHPEG) has developed a specific genetics core competency for health professionals for incorporating the use of information technology [15]. According to the NCHPEG's core competency 2.4, health professionals are encouraged to "use information technology to obtain credible, current information about genetics" [15; paragraph 2.4]. Apps are one of the important avenues. Of note, this study is also useful for researchers, the lay public, and health professional students, as they may be genetics/genomics app current or potential users, those who work with clients who are genetics/genomics app users, and those who are interested in app studies.

Our study has several important implications. Although there were 88 genetics/genomics apps, only about a quarter of the evaluated apps were developed by credible or authoritative entities, such as universities, hospitals, professional organizations, government, and NGOs. In addition, because of the inadequate number of reviewers, over one-third of the genetics/genomics apps did not have a customer rating. Lack of customer rating may prevent future app users from evaluating or reviewing the apps prior to downloading. Caution must be taken for both health professionals and consumers when using those apps.

While the majority of the apps served as references or resources (i.e., providing general genetics/genomics information and/or tutorials), some apps provided lifestyle recommendations to the general public, mostly based on the DTC genetic test results. Specifically, using integrated schemes and algorithms, those apps could provide an interpretation of genetic test results and then offer personalized recommendations regarding nutrition and physical activity. Nevertheless, although those apps have the potential to promote healthy behaviors based on genetic information, the accuracy and evidence of their recommendations are unclear. Therefore, it is important to engage geneticists and genetic counselors to access the app users' medical records to verify the genetic testing results and relevant recommendations as well as provide genetic counseling and necessary education.

The average MARS score was 3.78 out of five for all 88 genetics/genomics apps reviewed in this study. The overall quality of the apps was only slightly above average, suggesting a possible need for improvements. Specifically, while the functionality score was overall adequate among the apps, the app information scale had the lowest score among the four MARS scales. A potential explanation may be the low scores of the evidence-based item, which suggested that the app had not been trialed/tested and/or that 
the results had not been published in scientific journals. Another issue associated with the low app information score may be the inadequate credibility (i.e., uncertainty of the trustworthiness of the source). As such, healthcare professionals should examine genetics/genomics apps thoroughly when using them, especially for the apps that have clinical implications. Authoritative agencies, such as the FDA, MDD, and MHRA, can also ensure the credibility and evidence-based verification of the genetics/genomics apps through the development and implementations of relevant regulations and guidelines.

Similar to the overall MARS score, the average app engagement score for all the reviewed genetics/genomics apps was merely slightly above average (3.69 out of five points). Although four of the five items (i.e., entertainment, interest, interactivity, and target group) yielded adequate scores, app customization had a fairly low score ( 2.43 out of five points). This finding suggests that the genetics/genomics apps generally had insufficient settings for the users to adjust their preferred features, such as sound and notifications. Poor engagement design might discourage the users to utilize those apps frequently, which might jeopardize the purpose of the genetics/genomics apps. Thus, apps should contain a wider range of setting options for users to modify the apps based on their needs and preferences. In addition, the aesthetics score was also only slightly above average (3.70 out of five), particularly in graphics and visual appeal items. Therefore, more appealing, aesthetic genetics/genomics apps with improved resolution, color, style, memorability, and professionalism of the graphics and visual design may need to be developed to engage users in the future.

There are four main limitations in this study. First, because apps are constantly updating, it is possible that some of the included genetics/genomics apps have changed their names and/or were no longer available on Apple Store or Google Play. Second, we considered only apps in English. Thus, there may be some non-English genetics/genomics apps that were not in this review. Third, we included apps from Apple and Android systems in this review, as these are the two largest operating systems for apps. However, there may be a few other genetics/genomics apps on Amazon and Windows platforms. Fourth, the MARS does not include a question/item that specifically assesses the citations or references for the app content. As this information can help evaluate the quality of the app information, such measure should be included in future research and review.

Despite the limitations, this study holds several strengths. In particular, this is the first study, to the best of our knowledge, to identify the existing genetics/genomics apps and summarize their characteristics. Our search was extensive by including apps from both Apple and Android as well as both paid and unpaid apps. We found 88 available genetics/genomics apps and summarized their characteristics. The majority of the apps (1) did not have reliable or authoritative agencies as developers, such as universities or hospitals, (2) had Apple based operating system, (3) had reference/resource as an app feature, (4) required a web access to function, (5) did not focus on specific diseases, and (6) targeted health professional students as their audience. Furthermore, and most importantly, beyond merely presenting the characteristics of the genetics/genomics apps, each app's quality was evaluated using a validated scaleMARS. We found that the quality of the genetics/genomics apps overall still has much room for improvement, especially in the credibility and evidence-based items of app information as well as the customization items of app engagement. Accordingly, genetics/genomics apps might provide a promising avenue to genetics/genomics resources as well as health promotion and clinical tools. Due to the concerns regarding the quality of the apps overall, however, caution must be taken for researchers, health professionals and students, and the lay public when using those apps. Moreover, refining the existing genetics/genomics apps and creating new ones with a higher quality is strongly needed to fulfill the purposes of those apps and better serve the users.

\section{Compliance with ethical standards}

Conflict of interest The authors declare that they have no conflict of interest.

Publisher's note: Springer Nature remains neutral with regard to jurisdictional claims in published maps and institutional affiliations.

\section{References}

1. Statista. Cumulative number of apps downloaded from the Apple App Store from July 2008 to June 2017 (in billions). Statista, Inc.; 2018. https://www.statista.com/statistics/263794/ number-of-downloads-from-the-apple-app-store/. Accessed 24 Jul 2018.

2. Statista. Cumulative number of apps downloaded from the Google Play as of May 2016 (in billions). Statista, Inc.; 2018. https://www.statista.com/statistics/281106/number-of-android-a pp-downloads-from-google-play/. Accessed 24 Jul 2018.

3. Fox S, Duggan M. Pew internet project: mobile health 2012. Washington, DC: Pew Research Center's Internet \& American Life Project (US); 2012. http://www.pewinternet.org/files/oldmedia/Files/Reports/2012/PIP_MobileHealth2012_FINAL.pdf. Accessed 24 Jul 2018.

4. Kreb P, Duncan DT. Health app use among US mobile phone owners: a national survey. JMIR Mhealth Uhealth. 2015;3:e101.

5. Santo K, Richtering SS, Chalmers J, Thiagalingam A, Chow CK, Redfern J. Mobile phone apps to improve medication adherence: a systematic stepwise process to identify high-quality apps. JMIR Mhealth Uhealth. 2016;4:e132.

6. Molteni M. Wellness apps evade the FDA, only to land in court. Boone (IA): CNMN Collection (US); 2017. https://www.wired. 
com/2017/04/wellness-apps-evade-fda-land-court/. Accessed 24 Jul 2018.

7. European Committee for Electrotechnical Standardization. FAQs. Bruxelles, Belgium: European Committee for Electrotechnical Standardization; 2018. https://www.cenelec.eu/faq/faq_entry.htm. Accessed 2 Nov 2018.

8. Medicines and Healthcare products Regulatory Agency. Medical devices: conformity assessment and the CE mark. London, UK: Government Digital Service (UK); 2016 [cited 2018 Nov 28]. Available from: https:/www.gov.uk/guidance/medical-devicesconformity-assessment-and-the-ce-mark.

9. Medicines and Healthcare products Regulatory Agency. Register as a manufacturer to sell medical devices. London, UK: Government Digital Service (UK); 2018. https://www.gov.uk/guida nce/register-as-a-manufacturer-to-sell-medical-devices\#ce-ma rking. Accessed 28 Nov 2018.

10. Armstrong S. Which app should I use? Br Med J. 2015;351:h4597.

11. Wilson H, Stoyanov SR, Gandabhai S, Baldwin A. The quality and accuracy of mobile apps to prevent driving after drinking alcohol. JMIR Mhealth Uhealth. 2016;4:e98.

12. Wolf JA, Moreau JF, Akilov O, et al. Diagnostic inaccuracy of smartphone applications for melanoma detection. JAMA Dermatol. 2013;149:422-6.

13. National Human Genome Research Institute. The human genome project completion. Bethesda (MD): National Human Genome Research Institute (US); 2010. https://www.genome.gov/ 11006943/human-genome-project-completion-frequently-askedquestions/. Accessed 24 Jul 2018.

14. Miller R, Khromykh A, Babcock H, Jenevein C, Solomon BD. Putting the pieces together: clinically relevant genetic and genomic resources for hospitalists and neonatologists. Hosp Pediatr. 2017;7:108-14.

15. National Coalition for Health Professional Education in Genetics. Core competencies for all health professionals. Bar Harbor (ME): The Jackson Laboratory (US); 2007. https://www.jax.org/educa tion-and-learning/clinical-and-continuing-education/ccep-non-ca ncer-resources/core-competencies-for-health-care-professionals. Accessed 24 Jul 2018.

16. Boyce N. The lancet technology: September, 2011. Lancet. 2011;378:975.

17. Myers RB, Millman B, Noor MA. Genetics and evolution: an iOS application to supplement introductory courses in transmission and evolutionary genetics. G3: Genes, Genomes, Genet. 2014;4:779-81.

18. Bender J, Yue R, To M, Deacken L, Jadad A. A lot of action, but not in the right direction: systematic review and content analysis of smartphone applications for the prevention, detection, and management of cancer. J Med Internet Res. 2019;15:e287.
19. Arnhold M, Quade M, Kirch W. Mobile applications for diabetics: a systematic review and expertbased usability evaluation considering the special requirements of diabetes patients age 50 years or older. J Med Internet Res. 2014;16:e104.

20. Chomutare T, Fernandez-Luque L, Arsand E, Hartvigsen G. Features of mobile diabetes applications: review of the literature and analysis of current applications compared against evidencebased guidelines. J Med Internet Res. 2011;13:e65.

21. Huguet A, Rao S, McGrath PJ, et al. A systematic review of cognitive behavioral therapy and behavioral activation apps for depression. PLoS ONE. 2016;11:e0154248.

22. Conroy DE, Yang C, Maher JP. Behavior change techniques in top-ranked mobile apps for physical activity. Am J Prev Med. 2014;46:649-52.

23. Nicholas J, Larsen ME, Proudfoot J, Christensen H. Mobile apps for bipolar disorder: a systematic review of features and content quality. J Med Internet Res. 2015;17:e198.

24. Stoyanov SR, Hides L, Kavanagh DJ, Zelenko O, Tjondronegoro $\mathrm{D}$, Mani M. Mobile app rating scale: a new tool for assessing the quality of health mobile apps. JMIR Mhealth Uhealth. 2015;3:e27.

25. Bardus M, van Beurden SB, Smith JR, Abraham C. A review and content analysis of engagement, functionality, aesthetics, information quality, and change techniques in the most popular commercial apps for weight management. Int J Behav Nutr Phys Act. 2016;10:35.

26. Grainger R, Townsley H, White B, Langlotz T, Taylor WJ. Apps for people with rheumatoid arthritis to monitor their disease activity: a review of apps for best practice and quality. JMIR Mhealth Uhealth. 2017;5:e7.

27. Creber RMM, Maurer MS, Reading M, Hiraldo G, Hickey KT, Iribarren S. Review and analysis of existing mobile phone apps to support heart failure symptom monitoring and self-care management using the Mobile Application Rating Scale (MARS). JMIR Mhealth Uhealth. 2016;4:e74.

28. DiFilippo KN, Huang W, Chapman-Novakofski KM. A new tool for nutrition app quality evaluation (AQEL): development, validation, and reliability testing. JMIR Mhealth Uhealth. 2017;5: e163.

29. Goldgar C, Michaud NP, Jenkins J. Physician assistant genomic competencies. J Physician Assist Educ. 2016;27:110-6.

30. Howington L, Riddlesperger K, Cheek DJ. Essential nursing competencies for genetics and genomics: implications for critical care. Crit Care Nurse. 2011;31:e1-e7.

31. Centers for Disease Control and Prevention's Office of Public Health Genomics. Genomic workforce competencies. Atlanta (GA): Centers for Disease Control and Prevention (US); 2010. https://www.cdc.gov/genomics/translation/competencies/. Accessed 24 Jul 2018. 